\title{
Grade 4 oral mucositis and prolonged pancytopenia with high dose intravenous methotrexate in a patient of philadelphia positive acute lymphoblastic leukemia
}

\author{
Vidisha M. Shah $^{a}{ }^{a}$, Dimple S. Mehta ${ }^{a}$
}

\author{
${ }^{a}$ Department of Pharmacology, \\ C.U. Shah Medical College, \\ Surendranagar 363001, India \\ Received: 28 July 2012 \\ Revised: 7 August 2012 \\ Accepted: 9 August 2012 \\ *Correspondence to: \\ Dr. Vidisha M. Shah, \\ Email: \\ Vidisha_pharmac@yahoo.com
}

\begin{abstract}
Antimetabolite drugs are rarely associated with grade 3 or 4 mucositis. However specific side effects such as bone marrow suppression, pancytopenia, hospitalization, high cost limit the use of intravenous methotrexate. Here we report a case of 25-year-old girl patient with Acute Lymphoblastic Leukemia (ALL) treated with 24 hour methotrexate in her continuous phase of chemotherapy. After 12 hours of completing the treatment she developed oral mucosal pain \& she was not able to speak. After a week, her White Blood Cell (WBC) count was 600/cumm with fever and she could not speak and eat or drink. So she was hospitalized for few days till she can drink something. We report high grade mucositis with methotrexate. Hematologists should be aware of this possible side effect to undertake early intervention.
\end{abstract}

Keywords: Methotrexate (MTX), Mucositis, Pancytopenia, ALL

\section{INTRODUCTION}

Methotrexate has infrequently caused serious (sometimes fatal) side effects. Therefore, this medication should be used only to treat cancer and other severe diseases caused by an overactive immune system. ${ }^{1}$ This medication is used to treat trophoblastic neoplasms, leukemia, breast cancer, psoriasis, osteosarcoma, mycosis fungoides, psoriatic arthritis, and a variety of solid tumors. ${ }^{2}$ The most frequently reported adverse reactions include ulcerative stomatitis, leukopenia, nausea, and abdominal distress. Other frequently reported adverse effects are malaise, undue fatigue, chills and fever, dizziness and decreased resistance to infection. ${ }^{3}$ One of the main secondary toxic side effects of antimitotic agents used to treat cancer patients is intestinal mucositis. This one is characterized by compromised digestive and absorptive functions, barrier integrity, and immune competence. ${ }^{4}$ Clinical evidence from patients undergoing Methotrexate (MTX) therapy indicates that personal response to MTX is different. Some people display minor side effects, while others suffer from severe adverse effects that lead to the discontinuation of cancer therapy.
Mucositis is the painful inflammation and ulceration of the mucous membranes lining the digestive tract, usually as an adverse effect of chemotherapy and radiotherapy treatment for cancer. ${ }^{5}$ Mucositis usually appears along the entire gastrointestinal tract from mouth to anus and causes general debility. Mucositis of the intestine is characterized by increased crypt apoptosis and villus atrophy, leaving the mucosal tissue open to ulceration and infection. ${ }^{6-8}$

For most cancer treatment, about $5-15 \%$ of patients get mucositis. Oral mucositis also represents a major nonhematologic complication of cytotoxic chemotherapy and radiotherapy associated with significant morbidity, pain, odynodysphagia, dysgeusia, and subsequent dehydration and malnutrition. ${ }^{9}$ But severe (grade 3 or 4 ) mucositis due to MTX is a very rare finding.

\section{CASE REPORT}

We report a case of 25-year-old girl taking injectable methotrexate for Philadelphia positive Acute Lymphoblastic Leukemia (ALL). She was admitted to the hospital for her $1^{\text {st }}$ continuation therapy for ALL. She had already completed her induction phase. She was given high dose intravenous MTX (3 gm) diluted in normal saline over 20 hours infusion along with triple intrathecal 
chemotherapy (Intrathecal MTX, Ara-C and hydrocortisone). After 12 hours of completing the treatment, she started having anorexia and vomiting. She was not able to eat anything. Then she was given 3 doses of intravenous leucovorin and 3 doses of oral leucovorin 12 hourly. After a week, she got fever and could not even drink anything from mouth. Her WBC count was around $600 /$ cumm. So, she was admitted again in an isolation room in sterling hospital. She was diagnosed as having grade 4 mucositis with bone marrow suppression and pancytopenia. She was kept nil by mouth for few days. Higher antibiotics, parenteral nutrition and filgrastim were given.

On examination, her lips were swollen and black. Constant bleeding and salivation was there from her mouth, which was interfering her sleep also. That was constantly cleaned by gauze pieces. In the mouth, whitish membrane was found in all over the mouth specially both side on buccal mucosa. She was diagnosed as having fungal infection. She was passing blood in the stool for 34 days. On ultrasonography examination of abdomen, inflammation of intestinal mucosa was found. She had no complain of vomiting afterwards.

During 13 days of stay at hospital, WBC count was recorded daily. It was below 1000/cumm for 11 days. She was recovered on day 11 when the count was 1500 /cumm. She was allowed to drink and have soft diet. Still her lips were black and some whitish membranes were still there on buccal mucosa. Patient was discharged on day 13. Her condition was better. She could speak and eat soft diet.

\section{DISCUSSION}

Adverse Drug Reactions (ADRs) are a major hazard of modern chemotherapy. In routine clinical practice ADRs remain largely unrecorded even though it significantly affects quality of life.

Methotrexate, an antifolate agent, class of antimetabolite, is approved for the treatment of ALL. It inhibits dihydrofolate reductase-blocking the conversion of dihydrofolic acid to tetrahydrofolic acid which is required for purine synthesis and amino acid inter conversion. ${ }^{10}$ It acts by blocking DNA and RNA synthesis, inducing apoptosis, and slowing down or stopping cell proliferation. ${ }^{11}$ Since their actions are not specific to tumor tissues, they also induce deleterious effects on rapidly proliferating cells such as bone marrow and gut mucosa cells and produce mucositis. During experimental colitis, proinflammatory cytokines have been associated with serotonin release from the hypothalamic paraventricular nucleus and may induce a reduction of food intake. ${ }^{12}$ Low food intake is known to induce rapid and profound wasting of several organs, especially in the small intestine, which is more sensitive to food restriction due to its very high rate of protein turnover.
According to WHO, mucositis can be divided in 5 grades. Grade 0 (no changes), Grade 1 (Soreness with erythema) Grade 2 (Erythema, ulcers, can eat solids) Grade 3 (Ulcers, liquid diet only) Grade 4 (Alimentation not possible). ${ }^{13}$ Here, the patient's alimentation was not possible so we can consider it as grade 4 mucositis.

The bone marrow suppression may be treated with growth factors or even compensated by bone marrow transplantation. ${ }^{14}$ In contrast, there is no efficient standard treatment to reduce oral and gastrointestinal injuries called intestinal mucositis. Boukhettala et al study supports our short case.

\section{REFERENCES}

1. Methotrexate. Available at http://www.medicinenet.com/methotrexateinjection/article.html. Accessed 25 July 2012.

2. National Institutes of Health. Methotrexate injection. Available http://www.nlm.nih.gov/medlineplus/druginfo/meds/ a682018.html\#other-uses. Accessed on 25 July 2012.

3. Methotrexate side effects. Available at http://www.drugs.com/sfx/methotrexate-sideeffects.html. Accessed 25 July 2012.

4. Boukhettala N, Leblond J, Claeyssens S, Faure M, Le Pessot F, Bo^le-Feysot C, et al. Methotrexate induces intestinal mucositis and alters gut protein metabolism independently of reduced food intake. Am J Physiol Endocrinol Metab 2009; 296:E182E190.

5. Ridge JA, Glisson BS, Lango MN, et al. "Head and Neck Tumors" in Pazdur R, Wagman LD, Camphausen KA, Hoskins WJ (Eds). Cancer Management: A Multidisciplinary Approach. 11 ed. 2008.

6. Naidu MU, Ramana GV, Rani PU, Mohan IK, Suman A, et al. Chemotherapy-induced and/or radiation therapy-induced oral mucositiscomplicating the treatment of cancer. Neoplasia 2004; 6:423-31.

7. Sonis ST. The pathobiology of mucositis. Nat Rev Cancer 2004; 4:277-84.

8. Logan RM, Stringer AM, Bowen JM, Gibson RJ, Sonis ST, et al. Is the pathobiology of chemotherapy-induced alimentary tract mucositis influenced by the type of mucotoxic drug administered? Cancer Chemother Pharmacol 2009; 63:239-51.

9. Bitran JD, Samuel B, Klein L, Hanauer S, Johnson L, Martinec J, Harris E, Kempler J, White L. Random high dose chemotherapy supported by hematopoietic progenitor cells yields prolonged survival in stage IV breast cancer. Bone Marrow Transplant 1996; 17:157-62.

10. Tripathi KD. Antimetabolites. Essentials of Medical Pharmacology, $6^{\text {th }}$ ed. New Delhi: Jaypee brothers; 2008:823. 
11. Keefe DM, Brealey J, Goland GJ, Cummins AG. Chemotherapy for cancer causes apoptosis that precedes hypoplasia in crypts of the small intestine in humans. Gut 2000; 47:632-7.

12. Ballinger A, El-Haj T, Perrett D, Turvill J, Obeid O, Dryden $\mathrm{S}$, et al. The role of medial hypothalamic serotonin in the suppression of feeding in a rat model of colitis. Gastroenterology 2000; 118:54453.

13. Oral Mucositis. Available at http://painconsortium.nih.gov/symptomresearch/cha pter_17/sec7/cghs 7table71pop1.htm. Accessed on 25 July 2012.

14. Muscaritoli M, Grieco G, Capria S, Iori AP, Rossi Fanelli F. Nutritional and metabolic support in patients undergoing bone marrow transplantation. Am J Clin Nutr 2002; 75:183-90. 